\title{
Villa Mairea. El racionalismo nórdico de Alvar Aalto
}

\section{Giuliana Godoy Vera}

Universidad de Lima, Perú

Recibido: 16 de mayo de 2014 / Aprobado: 11 de julio de 2014

El presente análisis aborda una de las obras más representativas de la vivienda unifamiliar moderna: la Villa Mairea, del arquitecto nórdico Alvar Aalto. Esta obra es un excelente ejemplo de una modernidad que busca raíces locales y que no pretende ser parte de un estilo internacional, a pesar del reconocimiento mundial de la obra y del autor. Se analiza el trabajo del espacio, la materialidad y la adaptación a un clima y ubicación específicos. Las tres partes del artículo buscan guiar la lectura dentro de las principales variables de la obra, para concluir en su importancia como obra trascendental de mitad del siglo XX, pero con proyección hasta nuestros días.

arquitectura, Villa Mairea, Alvar Aalto, arquitectura moderna, siglo XX

\section{Villa Mairea. The nordic rationalism of Alvar Aalto}

This analysis addresses one of the most representative works of modern single house: Villa Mairea from the nordic architect Alvar Aalto. This work is an excellent example of a modernity that seeks local roots and is not intended to be part of an international style, despite the international recognition of the work and its author. Work of space, materiality, and adaptation to a specific climate and location are analyzed. The three parts of the article seek to guide the reading among the most important variables of the work, concluding with its importance as a transcendent work of mid-twentieth century, but to this day projection.

architecture, Villa Mairea, Alvar Aalto, modern architecture, 20th century 


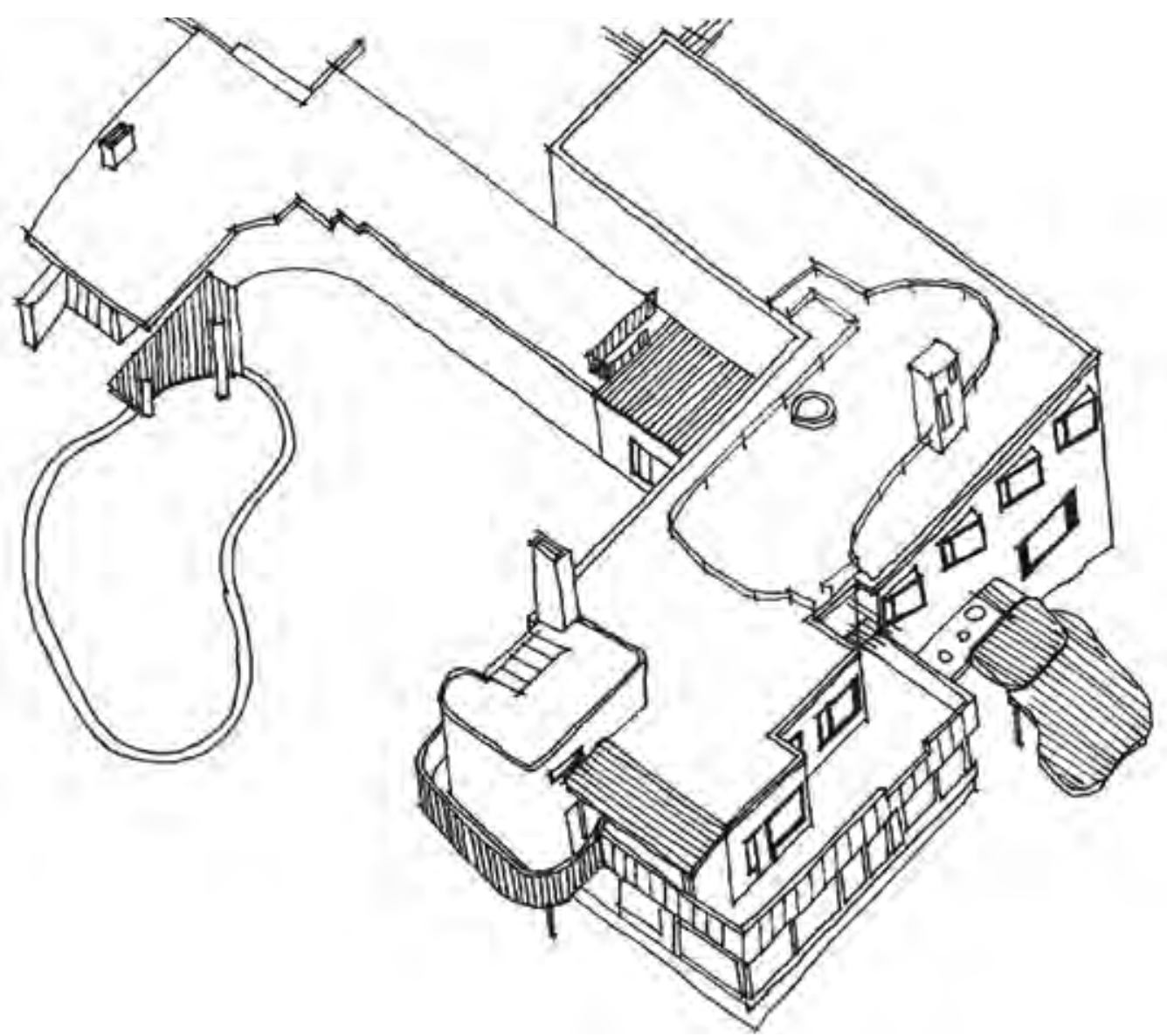

Figura 1.

Axonometría.

\section{INTRODUCCIÓN}

La Villa Mairea tiene varios caracteres innovadores y únicos; rige un principio de dualidad entre lo natural - teniendo en cuenta que el emplazamiento de la obra es un lugar aislado que forma parte de la naturaleza, con un paisaje donde abundan árbolesy lo construido; donde el espacio interior y el espacio exterior son parte de esta dualidad, que va de la mano con lo natural y lo artificial, respectivamente.

Los elementos presentes en toda la obra, desde las fachadas hasta la parte más privada, van transformándose conforme uno se adentra en los volúmenes y se aleja de la naturaleza, esto se aprecia en las formas y en los materiales que se han utilizado.La piscina tiene una forma orgánica de trazado irregular, al igual que la cubierta de la entrada principal y los escalones que nos llevan al ingreso.
Como dice Frampton, "Se combina y representa una mezcla de la nueva corriente modernista del siglo XX y el movimiento romántico-nacionalista" (2010, p. 201).

Por la parte regionalista, al ser Finlandia el país donde se desarrolló el proyecto, trata de formar parte del paisaje y no imponerse, lo llega a hacer en la medida de lo posible, pese a que también existen trazados ortogonales en sus volúmenes, logra mimetizarse con los altos árboles en sus elementos verticales que son las columnas y en la fachada con elementos repetitivos que siguen un ritmo y que a su vez van marcando el cerramiento según la distancia con la que se encuentren entre ellos; y así evocar con su arquitectura la naturaleza. Las columnas son importantes y varían en las diferentes estancias; algunas son simples, otras 


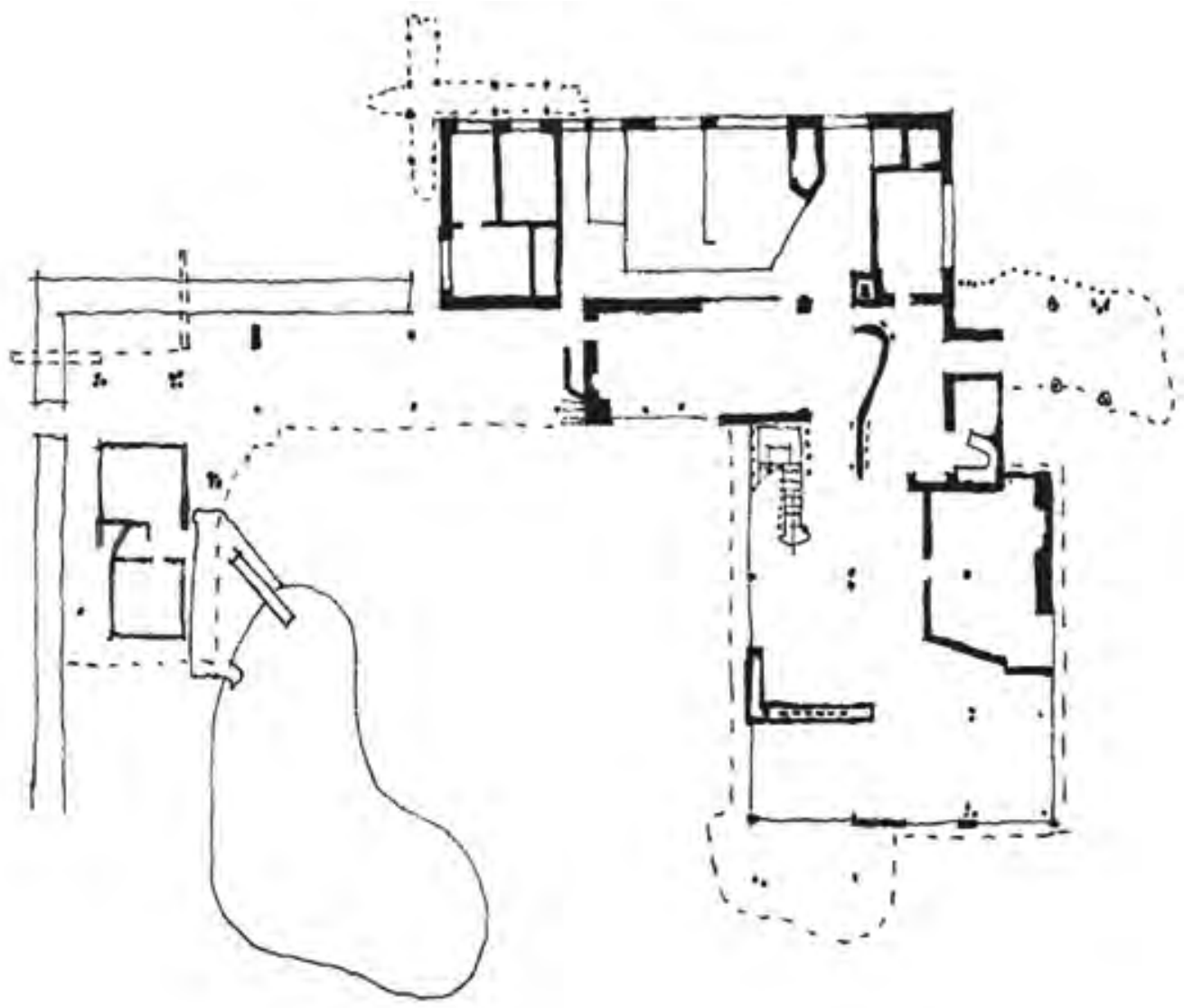

Figura 2

Planta del primer nivel.

dobles, con algún tipo de recubrimiento, y otras en forma de árbol; no todas cumplen con una función estructural. Todo está muy bien pensado. Y una vez que supe quién lo había diseñado estaba más segura de la decisión, porque Alvar Aalto es un destacado representante del modernismo y considero que la arquitectura que él ha creado está muy bien planteada y diseñada de manera inteligente; además, cuenta con todas las variables que necesitan ser consideradas en sus obras, sin abandonar su visión como arquitecto.

\section{ANÁLISIS CRÍTICO}

En el siglo XIX aparecieron nuevos materiales e innovaciones que revolucionaron la arquitectura de la época, como fueron el acero, el concreto y el ascensor. Los dos primeros fueron fundamentales para la construcción de edificios de gran resistencia $\mathrm{y}$ sin que ocupen demasiado espacio, y donde la estructura y los soportes no tengan que ser tan anchos ni obtener pequeñas luces libres, sino que las columnas o muros portantes podían separarse sin fragmentar de manera indeseada los espacios, dando mayor libertad al arquitecto al idear una obra y tener la posibilidad de una planta libre, cuya característica es una de las bases de la arquitectura moderna. Hay un gran cambio respecto de lo antiguo y tradicional.

La Villa Mairea es una obra de arquitectura moderna, porque se usa el concreto como un elemento que ayuda a crear un edificio sin grandes masas portantes que lo interrumpan y tiene planta libre, sin lo cual no sería capaz de haber grandes espacios en las áreas comunes. "Aalto pasa un momento de transición, de 


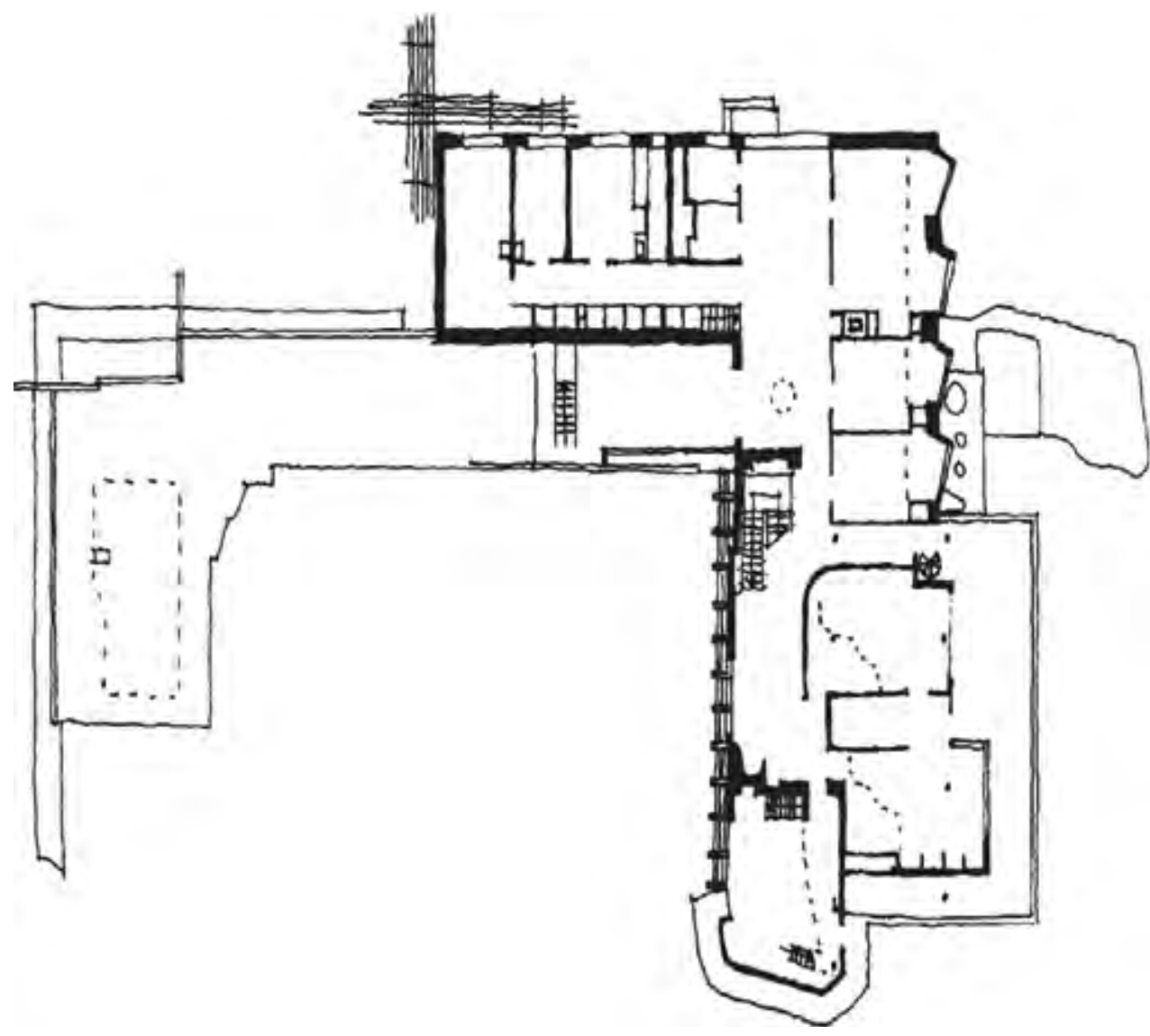

Figura 3.

Planta del segundo nivel.

estar ligado al funcionalismo en su anterior obra que fue el Sanatorio de Paimo, a 5 años después empezar a mezclarlo con su herencia nacionalista finlandesa" (Frampton, 2010, p. 203).

Comprende que las bases de la arquitectura moderna que se habían ido formando no tenían que ser limitantes, sino que eran unas herramientas para ser estudiadas y, dependiendo de cada proyecto, podían ser adaptadas, y como la obra era hecha en Finlandia se adoptaron rasgos regionalistas imperantes en las diferentes construcciones de este país, como el uso de la madera, y la relación interior-exterior tan necesaria para obtener un contacto directo con la naturaleza que rodeaba el emplazamiento del proyecto.

A pesar de los diferentes contrastes presentes en sus obras: modernidad-tradición, naturaleza- arquitectura, y de plantearse conjuntamente estas diferentes concepciones de lo que sería la Villa Mairea, esta existe como una unidad, donde las partes trabajan como un todo haciéndola más interesante y sin sacrificar nunca los rasgos importantes en su arquitectura, por la concepción tan cerrada y extrema que algunos tenían del racionalismo en los inicios del movimiento moderno.

\section{CONCLUSIONES}

Esta obra constituye unimportante aporte conceptual arquitectónico y muestra una alternativa diferente, que siguiendo al modernismo marca variantes sobre este, combinándolo con otras características, como el regionalismo, enriqueciéndolo con la 


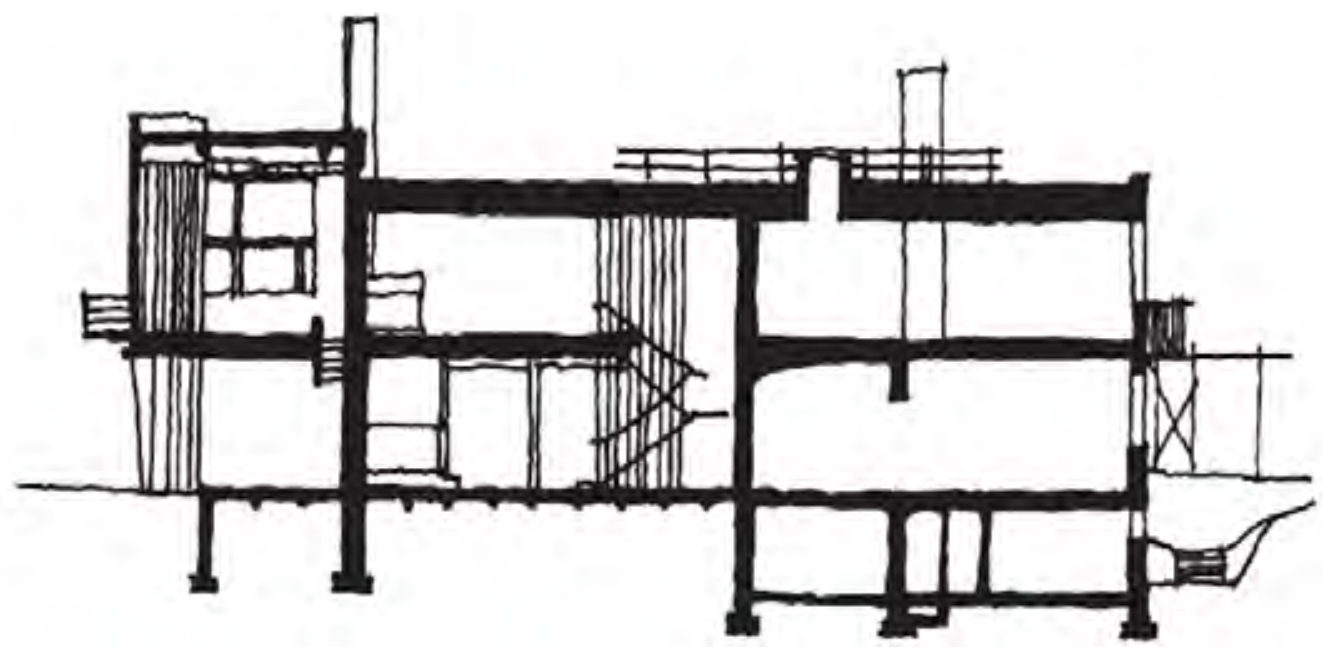

Figura 4

Sección.

introducción de un novedoso uso de materiales y la reinterpretación de técnicas de construcción tradicionales; a su vez, considera que la historia de la arquitectura de siglos anteriores no deja de tener importancia, pues es imposible no tomarla en cuenta en el futuro y revalorar el pasado, no por ser tradicional sino reinventándolo para seguir avanzando.

Existe una reconciliación entre los diferentes contrastes presentes en la obra, que son la modernidad con la tradición y la naturaleza con la arquitectura. Todo este cambio se ve plasmado en el edificio; los elementos utilizados, materiales, volúmenes, formas, etcétera, muestran la evolución de la que ha sido parte Alvar Aalto. Si nos fijamos en las obras que este arquitecto hizo antes y después de laVilla Mairea se distingue un punto de quiebre; aunque, en realidad, más que un punto de quiebre en sí es un punto de transición de un racionalismo industrialista a uno más humanista.

\section{REFERENCIAS}

Domínguez, L. A. (2003). Alvar Aalto, una arquitectura dialógica. Barcelona: Edicions UPC.

Filler, M. (2012). La arquitectura moderna y sus creadores: de Frank Lloyd Wright a Frank Gehry. Barcelona:Alba.

Frampton, K.(2010). Historia crítica de la arquitectura moderna. Barcelona: Gustavo Gili.

Lisnovsky, M. (2009). Aalto, La Villa Mairea y la refracción de la Arquitectura Moderna. 
Urbanismo.com. Recuperado de http://www. urbanismo.com/arquitecturayurbanismo/ aalto-la-villa-mairea-y-la-refraccion-de-laarquitectura-moderna/
Mujica, A. (2012). Arquivideo 3 -Villa Mairea por AlvarAalto.ApuntesdeArquitectura.Recuperado de http://apuntesdearquitecturadigital.blogspot. com/2012/09/arquivideo-3-villa-maireapor-alvar.html 